\title{
Unified Communications for Space Inventory Management
}

\author{
Kevin K. Gifford ${ }^{1}$ \\ UC, Boulder, CO, Zip Code \\ Patrick W. Fink ${ }^{2}$, Richard Barton ${ }^{3}$, and Phong H. Ngo ${ }^{4}$ \\ NASA Johnson Space Center, Houston, TX, 77058
}

\section{Introduction}

$\mathrm{T}$ o help assure mission success for long-duration exploration activities, NASA is actively pursuing wireless

technologies that promote situational awareness and autonomy. Wireless technologies are typically extensible, offer freedom from wire tethers, readily support redundancy, offer potential for decreased wire weight, and can represent dissimilar implementation for increased reliability. In addition, wireless technologies can enable additional situational awareness that otherwise would be infeasible. For example, addition of wired sensors, the need for which might not have been apparent at the outset of a program, might be extremely costly due in part to the necessary routing of cables through the vehicle.

RFID, or radio frequency identification, is a wireless technology with the potential for significant savings and increased reliability and safety in space operations. Perhaps the most obvious savings relate to the application of inventory management. A fully automated inventory management system is highly desirable for long-term sustaining operations in space environments. This assertion is evidenced by inventory activities on the International Space Station, which represents the most extensive inventory tracking experience base in the history of space operations. In the short term, handheld RFID readers offer substantial savings owing to reduced crew time for inventory audits. Over the long term, a combination of improved RFID technology and operational concepts modified to fully utilize the technology should result in space based inventory management that is highly reliable and requires very little crew time.

In addition to inventory management, RFID is likely to find space applications in real-time location and tracking systems. These could vary from coarse-resolution RFID portals to the high resolution afforded by ultra-wideband (UWB) RFID. Longer range RFID technologies that leverage passive surface acoustic wave (SAW) devices are being investigated to track assets on a lunar or planetary surface. For example, locating expended or surplus vehicles or equipment in a planetary salvage yard could be highly useful. The same technology is being evaluated for application in lunar road sign and lander navigation applications. In addition to enabling long range identification of assets, SAW-based passive RFID sensors also afford temperature telemetry. Other RFID sensor modalities are possible and are being investigated.

Middleware is increasingly recognized as a vital component in terrestrial RFID enterprise solutions. For a space vehicle or outpost, the middleware will be required to accommodate a large number of wired and wireless devices and networks, of which RFID systems constitute a subset. The middleware is also likely to fulfill many network security and quality-of-service requirements.

To evaluate the usefulness of various wireless technologies, the interoperability between wireless systems and devices, and the middleware that integrates these systems, we established a Lunar Habitat Wireless Test Bed (LHWT). Technologies proven in this environment are being transferred to other NASA analogs and incorporated in other R\&D projects. The LHWT also serves for demonstration of new technologies to NASA program and project managers.

\footnotetext{
${ }^{1}$ BioServe Space Technologies, CB 429, AIAA member.

${ }^{2}$ Deputy Chief, EM Systems Branch, 2101 NASA Pkwy/MS EV411

${ }^{3}$ Senior Staff Scientist, ERC Inc., NASA JSC, 2101 NASA Pkwy/MS EV814

${ }^{4}$ Electronics Engineer, EM Systems Branch, 2101 NASA Pkwy/MS EV411
} 


\section{RFID for Space-Based Inventory Management}

\section{A. Inventory Management for Space Applications - Present State}

Inventory management for flight applications requires a high degree of control, as improperly substituted items and early depletion of certain items can be catastrophic. Most short duration missions do not involve restocking, so resupply logistics are non-existent, but initial stocking and tracking of inventories is nonetheless quite important. For most long duration missions, resupply efforts are inherently complex, expensive, and infrequent. To date, the most extensive space-based inventory management operation has been the International Space Station (ISS). More detail on ISS inventory management, as well as a brief history of inventory management in human spaceflight, is provided below.

In early human spaceflight, such as the Apollo missions, inventories were kept on paper with diagrams showing inventory stowage locations. Even on NASA's Space Shuttle Orbiter, the crew is given hardcopy descriptions of item locations, without serial or model numbers. The Orbiter crew does have access to similar inventory information through an on-board laptop database, but additional assistance with item location is often required and entails radio communication with Mission Control.

On the International Space Station, approximately 20,000 items are tracked with the Inventory Management System (IMS) software application. Both flight and ground crews update the database daily. A handheld optical barcode reader is used to update the onboard database, and the IMS application performs complex updates. The ground and flight segment databases are synchronized by uplinking and downlinking "delta files". The common transport apparatus for smaller items is the Crew or Cargo Transfer Bag (CTB - see Fig. 1). Dimensions of the CTB are approximately 10in. x 17in. x 9.5in. The cargo ranges from crew clothing, to office supplies, pantry (food) items, and personal effects. The CTBs are packed on the ground, and like items within a CTB are usually stored in Ziploc bags. For some cargos, items are tracked both at the Ziploc bag level and at the individual item level. For other cargo types, tracking resolution extends only to the Ziploc bag level. In addition, optical barcode tags are also affixed directly to the CTBs.

In the 2008 timeframe, approximately 500 CTBs were onboard the ISS at any given time. The CTBs are typically stacked several deep and are often restrained by webbing or lines. Inventory audits required approximately 20 minutes per day for each crewmember. The time required to inventory a single CTB is also about 20 minutes. The process requires removal of each Ziploc bag and each tagged item, orienting the barcode to enable line-of-sight reading, and re-bagging the items. The process is greatly complicated by the zero-g environment, which requires extra care to prevent items from floating out of reach.

\section{B. Introduction of RFID for Space Applications: Handheld Readers}

To reduce the amount of crew time required for inventory audits on the ISS, NASA has been investigating handheld RFID readers as a replacement for the optical barcode readers. Although it is likely that initial readers would retain the optical barcode capability, it is anticipated that RFID would quickly become the preferred approach for audits. The handheld RFID reader will likely be the first step toward a crew-free inventory management system for later space vehicles. Testing has shown that the type of materials typically found in CTBs, and the density with which the materials are packed, often result in read accuracies less than $100 \%$ upon a single RFID interrogation of the CTB and contents therein. However, human assisted interrogation, in which the reader is scanned, or "painted", around the outside of the CTB, has been shown to be highly effective and efficient in auditing CTB contents, requiring about 20 seconds per CTB, compared to as much as 20 minutes per CTB using optical barcode technology. Furthermore, the handheld reader requires little infrastructure from the vehicle since it can operate on battery power and connectivity to a database can be provided by a wireless network. So, although it does not provide complete automation of inventory audits, the handheld reader does have the potential to greatly reduce the amount of time required of the crew. 


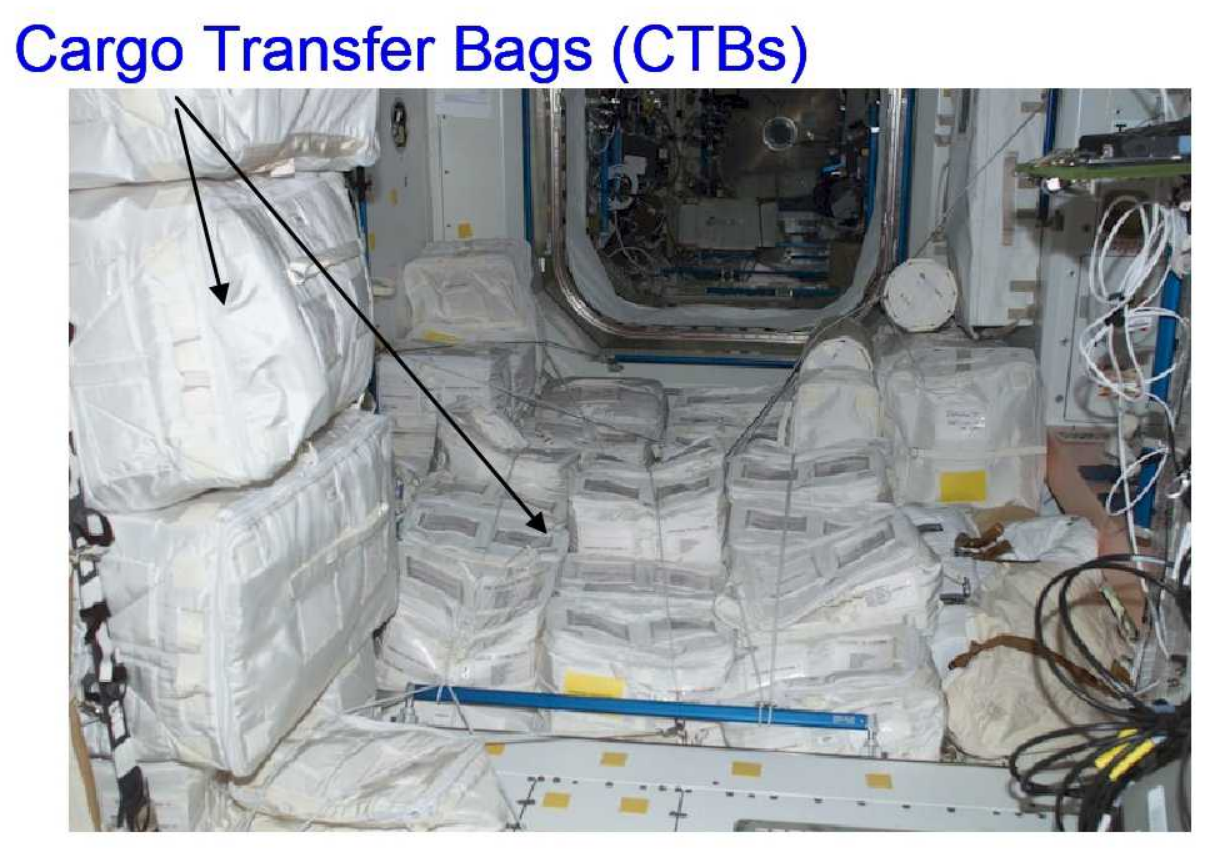

Figure 1. Cargo Transfer Bags on the International Space Station.

To determine the effectiveness of handheld RFID readers for crew-assisted inventory audits, CTBs and Ambulatory Medical Packs (AMPs) were scanned using four different handheld readers. These containers are shown in Fig. 2 and Fig. 3 below. For these tests, the pantry CTB was provisioned with personal hygiene items and office supplies. One RFID tag is placed on the outside of the CTB. Groups of similar items inside the CTB are placed in tagged Ziplock bags, and, in most cases, RFID tags are placed on individual items within the Ziplock bags as well. A total of 52 tags are associated with the CTB and its contents. The mixture of items inside the CTB includes metal foil bags and gel tubes, which can present difficulties for RFID interrogation. Different types of tags are applied at the item level based on previous testing of individual items.

The AMPs are similar to CTBs, although they are have a different shape (approximately $12.5 \mathrm{in} . \mathrm{x} 24.5 \mathrm{in} . \mathrm{x} 8 \mathrm{in}$.), and the contents comprise medical equipment and pharmaceuticals. For test purposes, controlled medications are substituted with pills and liquids that can be purchased over the counter. The test kit has 10 sub-packs containing 226 total tagged items For this test kit, most items have one RFID tag, but some items have two different RFID tags for evaluation. Unlike the CTB tests, pre-testing to optimize tag types for AMP items was not conducted, although such selection would likely improve read accuracy results, particularly for items with metallic or liquid content. For both the CTB and AMP tests, four different commercially available handheld interrogators were tested by five different individuals. Results corresponding to the CTB and AMP tests are shown in Table 1 and Table 2, respectively. Also shown is the transmit power for each reader. 

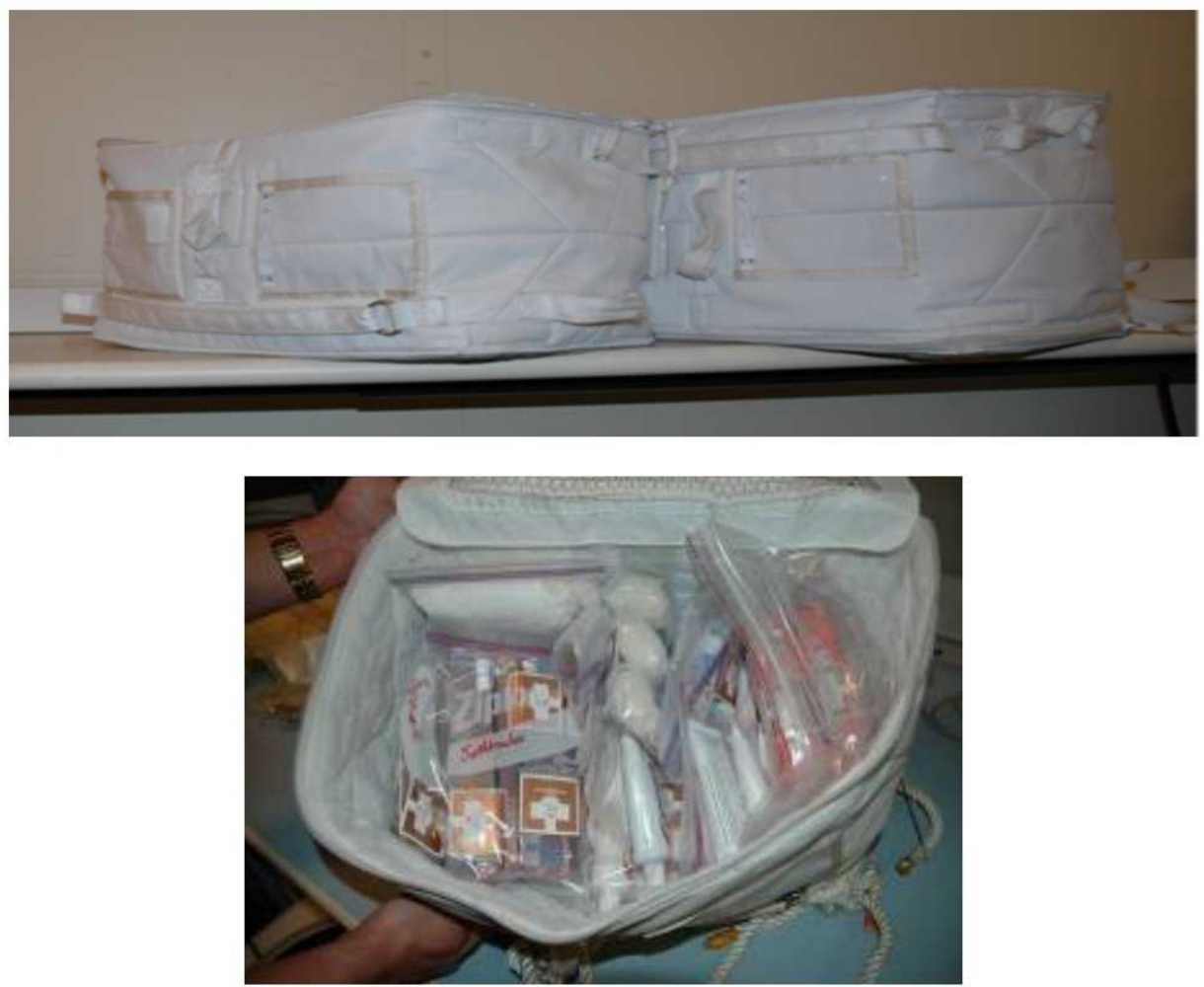

Figure 2. CTB level, Ziplock level, and item level RFID tags.
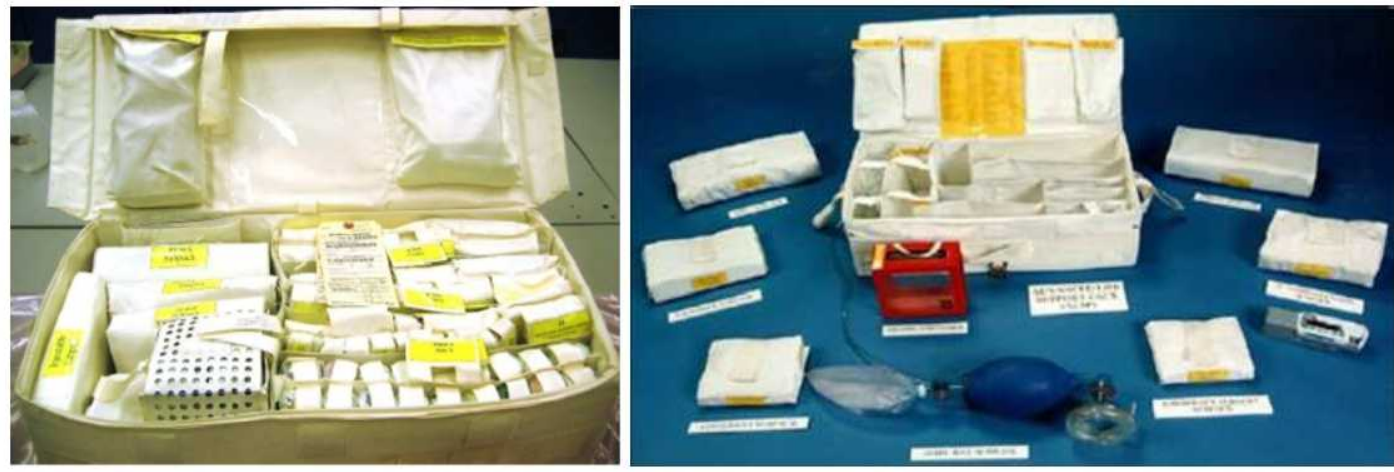

Figure 3. Ambulatory Medical Pack (AMP) and equipment/pharmaceuticals inside. 
Table 1. Number of CTB tags read by individuals (I1-I5) and \% of total tags read.

\begin{tabular}{|l|c|c|c|c|c|c|c|}
\cline { 2 - 8 } \multicolumn{1}{c|}{} & I1 & I2 & I3 & I4 & I5 & average & $\%$ \\
\hline Reader $130 \mathrm{dBm}$ & 48 & 48 & 49 & 48 & 48 & 48.2 & $92.7 \%$ \\
\hline Reader 2 $28 \mathrm{dBm}$ & 48 & 48 & 47 & 48 & 47 & 47.6 & $91.5 \%$ \\
\hline Reader 3 30 dBm & 42 & 42 & 44 & 45 & 43 & 43.2 & $83.1 \%$ \\
\hline Reader 4 30 dBm & 48 & 48 & 48 & 49 & 48 & 48.2 & $92.7 \%$
\end{tabular}

Table 2. Number of AMP tags read by individuals (I1-I5) and \% of total tags read.

\begin{tabular}{|l|c|c|c|c|c|c|}
\cline { 2 - 7 } \multicolumn{1}{c|}{} & I1 & I2 & I3 & I4 & average & $\%$ \\
\hline Reader $228 \mathrm{dBm}$ & 267 & 264 & 266 & 263 & 265 & $80.3 \%$ \\
\hline Reader $330 \mathrm{dBm}$ & 122 & 125 & 130 & 120 & 124.3 & $37.7 \%$ \\
\hline Reader $130 \mathrm{dBm}$ & 281 & 276 & 282 & 280 & 279.8 & $84.8 \%$ \\
\hline Reader $430 \mathrm{dBm}$ & 245 & 239 & 238 & 226 & 237 & $71.8 \%$ \\
\hline
\end{tabular}

\section{Portal-Based RFID for Space Applications}

Although use of the handheld reader promises to be a significant advancement in space-based inventory management, more automated methods are highly desirable due to the extreme demands on crew time. RFID portals within vehicles represent one possible step toward complete automation. Several key features are required for operational viability. A high read accuracy of tags is obviously one of these features. Another is low power consumption, which in turn drives a requirement for triggered operation. In addition, the direction of tags through the portal is highly desirable.

Performance with respect to the first two of these three was investigated by establishing RFID portals at each of two entrances of a lunar habitat mockup. Figure 4 shows two external antennas on the first portal of the mockup. Two additional antennas were placed on the interior of the entrance. A CTB with 52 tagged items was carried through the entrance using three configurations, which correspond to the CTB carried on the left side, in front, and on the right side of the human transporter. In addition, two trigger scenarios were employed. In the first scenario, the reader is "on" continuously as the CTB is carried toward and up the landing, starting from approximately 40 feet in front of the landing. In the second scenario, a pressure mat, placed five feet from the entrance, is used to trigger the reader. Each combination of carry configuration and trigger scenario was repeated five times. For all trials, a transmit power of $30 \mathrm{dBm}$ was used, and the four reader antennas were cycled sequentially.

Table 3 below shows the results for both item and Ziplock level read accuracies as a function of CTB position relative to the human transporter. Table 4 shows read accuracies for both continuous interrogation and pressure mat triggered scenarios. It can be seen that neither the CTB position nor the reader mode (triggered or continuous) had a significant effect on read accuracies. Results for tag directionality were under evaluation at the time of this publication. Initial attention is focused only on the sequence of first reads; that is, whether the interior or exterior portal antennas read the tags first. Planned follow-on work will also consider the timing of the triggered pressure mat. The requirement for read accuracies at the item and Ziplock bag level has yet to be determined, although the results achieved at the Ziplock level are likely to be sufficient. Improvements in RFID portal technology, supplemental RFID technologies, and modified operational scenarios will likely overcome the limitations currently presented by item level read accuracies reflected in these initial tests. 


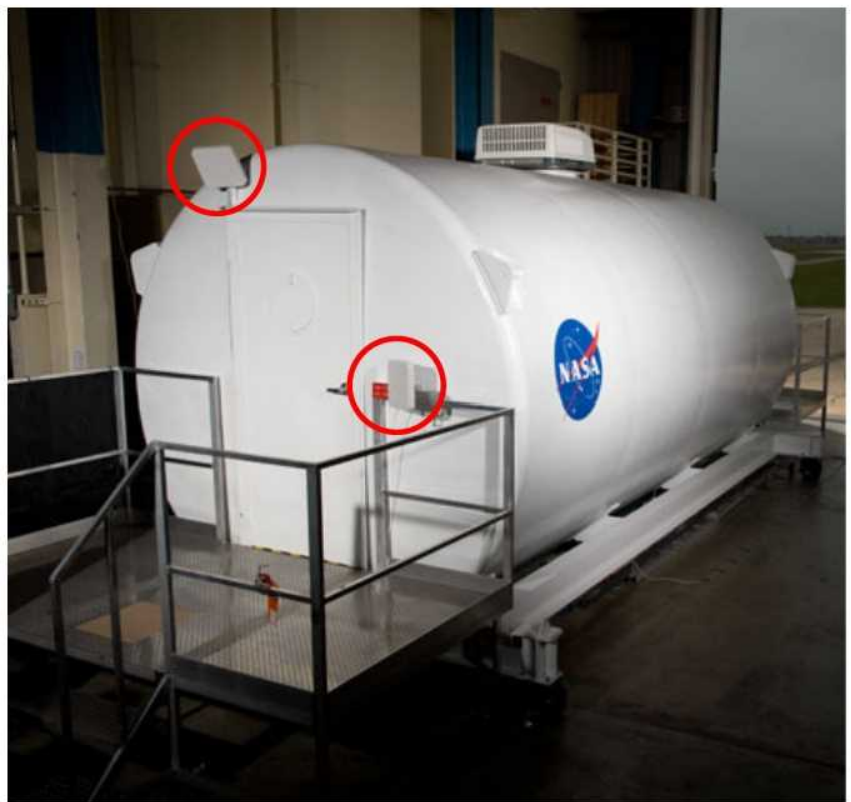

Figure 4. Lunar Habitat Wireless Test Bed (LHWT) with exterior RFID antennas (red circles) at first portal.

Table 4. Tag read accuracies as a function of CTB position relative to the human transporter.

\begin{tabular}{|l|c|c|c|}
\cline { 2 - 4 } \multicolumn{1}{c|}{} & Avg. front & Avg. right & Avg. left \\
\hline item level & $75.5 \%$ & $75.3 \%$ & $75.7 \%$ \\
\hline Ziplock level & $95.1 \%$ & $94.7 \%$ & $93.7 \%$ \\
\hline
\end{tabular}

Table 3. Tag read accuracies for triggered and continuous reader operation.

\begin{tabular}{|l|c|c|}
\cline { 2 - 3 } \multicolumn{1}{c|}{} & Avg. (triggered) & Avg. (continuous) \\
\hline item level & $76 \%$ & $75 \%$ \\
\hline Ziplock level & $95 \%$ & $94 \%$ \\
\hline
\end{tabular}

\section{D. "Smart" Shelves and Receptacles for RFID Space Applications}

Similar to terrestrial applications, densely packed containers with high metal or liquid content presents problems for RFID interrogation. "Smart" shelves and trash receptacles are examined as supplemental inventory tools to overcome some of these limitations. Versions presented here would not be directly suitable for zero-g environments, although zero-g implementations are in work. Both shelf and trash receptacle concepts support tag arrangements that are conducive to threshold-level electromagnetic field distributions in the vicinity of the tags. Figure 5 shows the relative locations for the RFID "Smart" shelves, the RFID trash receptacle, and soft cargo storage area.

Early tests with the "smart" shelf were conducted with medicinal containers and medical equipment, and results are indicated in Figure 7. For items that present difficulties for RFID interrogation, one concept being investigated is that of tracking item wrappers when discarded in a trash receptacle, as opposed to interrogation of items in their packed configurations. Testing with an RFID-enabled trash receptacle indicates near- $100 \%$ read accuracies for a wide variety of trash items, including items with metal and liquid content. Representative trash items include Ziploc bags, vacuum packed food items, conductive drink pouches, battery packs and various pharmaceuticals, both liquid and solid types. Figure 6 shows the trash receptacle and tagged contents within it. Some liquid containers 
were left entirely or partially full to represent partially consumed goods. Work on a zero-g version of the RFID trash receptacle is in progress.

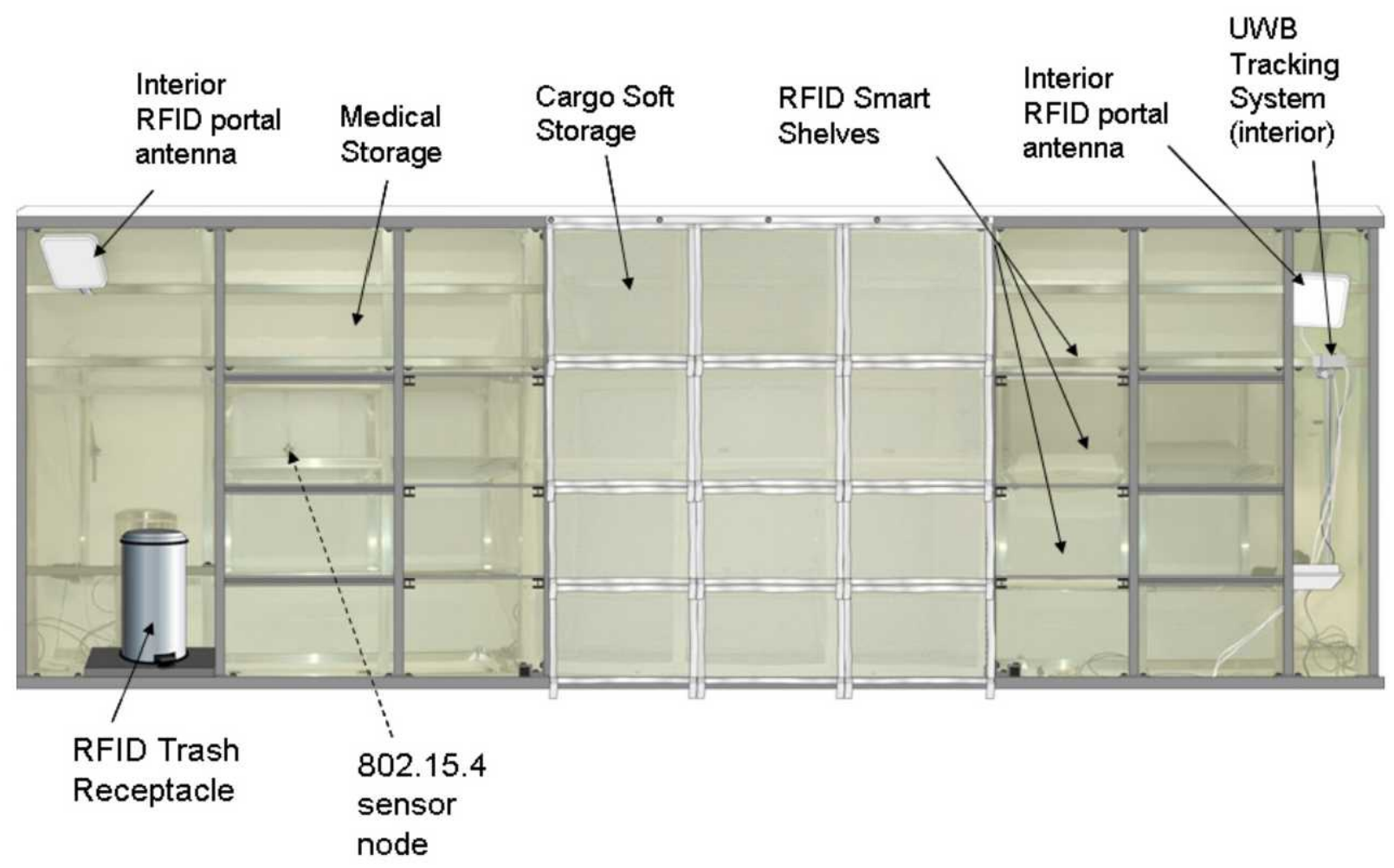

Figure 5. "Front" wall of I unar Habitat Wireless Test Bed and integrated RFID technologies.
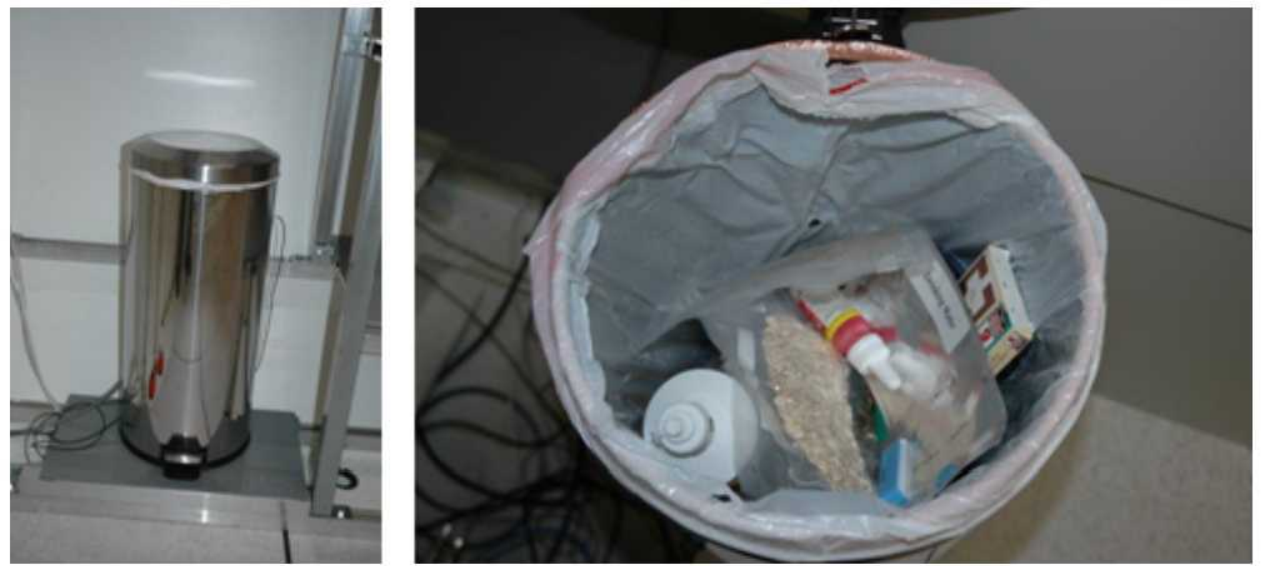

Figure 6. Non-zero-g RFID trash receptacle. 

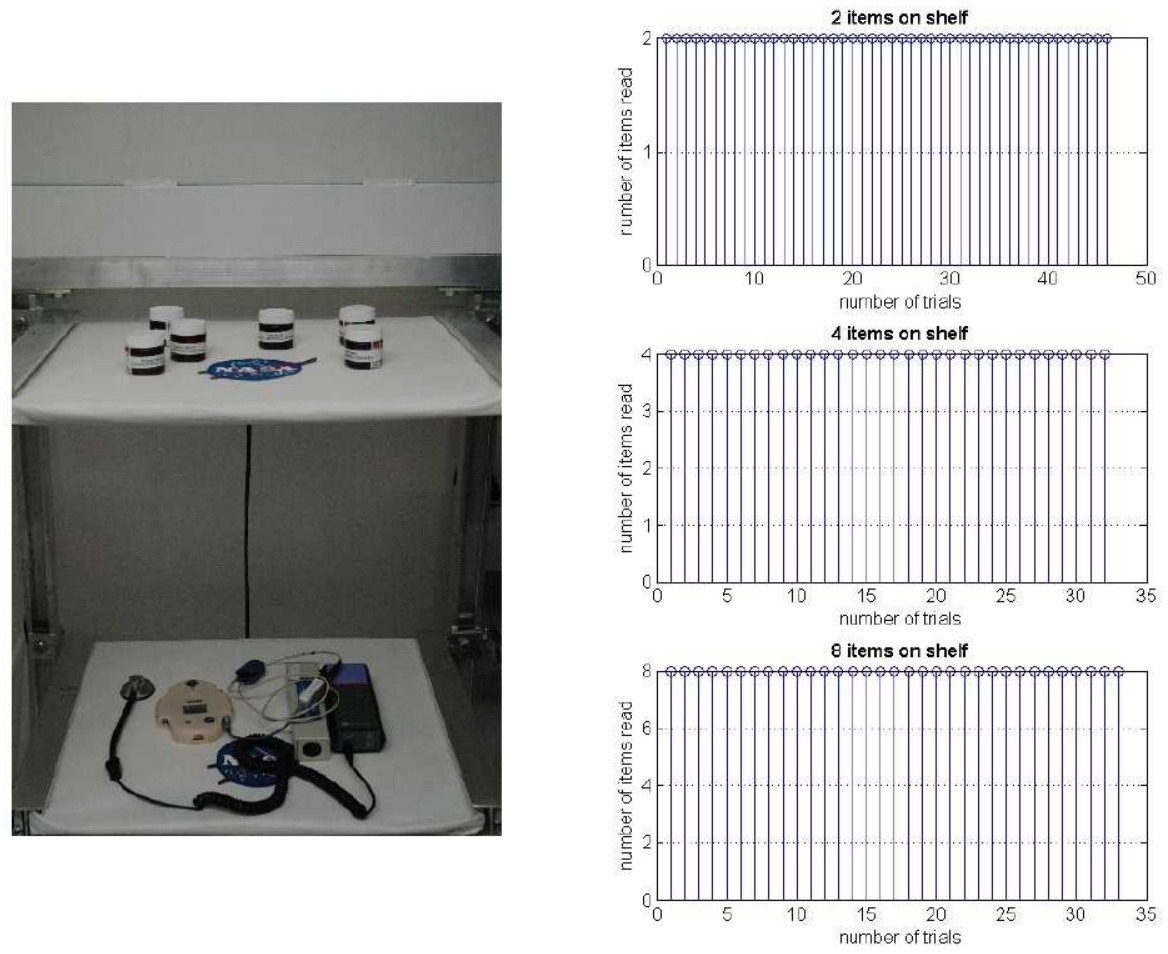

Figure 7. Items on RFID “smart" shelf and results indicating $100 \%$ read accuracy.

\section{RFID for Real-Time Location and Tracking Systems}

Another type of RFID technology, which relies on active rather than passive tags, is Ultra-Wideband or UWB RFID. UWB radio technology is a high-bandwidth communication scheme that offers several advantages for location estimation of targets based on radio-frequency emissions. In particular, UWB RFID tags transmit impulsive signals with sub-nanosecond duration and correspondingly high bandwidth of up to several GHz. As a result of the fine time resolution of the signals, UWB RFID tags are well-suited for precise positioning applications. In addition, the wide bandwidth of the signals results in very low power spectral densities, which reduces interference on other RF systems, and the short pulse duration reduces or eliminates pulse distortion (fading) and spurious signal detections due to multipath propagation. An example of a UWB signal is illustrated in Figure 10.

UWB RFID technology is often applied in Real-Time Location Systems (RTLS) that are utilized to identify the position of tagged equipment on demand in a dynamic environment such as a manufacturing facility or warehouse. An example of such a system is the Sapphire DART system developed by Multispectral Solutions, which has been integrated into the Lunar Habitat Wireless Testbed (LHWT) at JSC. The system consists of a processing hub connected to a set of UWB receivers which span the coverage area. The processing hub provides power, data, and synchronization to all receivers through a dedicated cabling infrastructure. The UWB receivers detect transmissions from active RFID tags attached to the assets being tracked. The tags are transmit-only devices that transmit a unique UWB pulse-train ID at a rate of up to one pulse train every second. The system is capable of $30 \mathrm{~cm}$ (1 foot) accuracy with read ranges of up to 90 meters and tag battery life in excess of 7 years with tags transmitting once per second.

The system is deployed in the LHWT as illustrated in Fig. 9. The figure shows the floor plan of the LHWT with the four UWB receivers, which are located near the ceiling of the habitat, circled in red. Also illustrated in the figure are the locations of three UWB RFID tags, shown as blue cross-hatched circles, as they would be displayed in the GUI of the UWB RFID tracking system implemented in the LHWT. A corresponding image of one of the UWB receivers mounted in the habitat is shown in Fig. 8. 


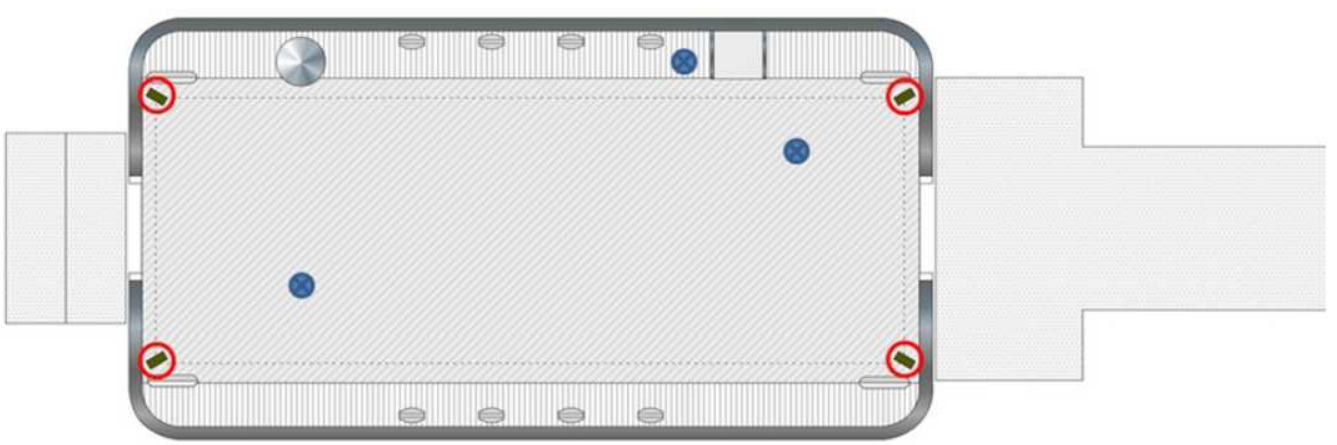

Figure 9. Floor plan of LHWT showing UWB receiver locations and three tag locations being tracked.
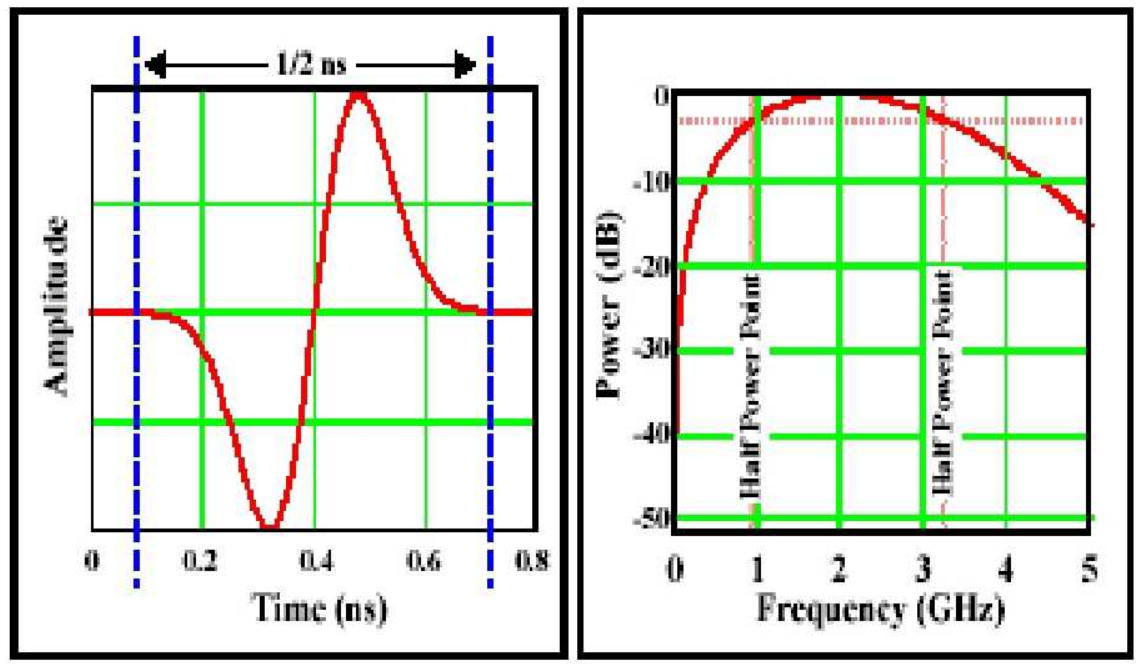

Figure 10. Illustration of UWB impulsive signal in time- and frequency-domains.

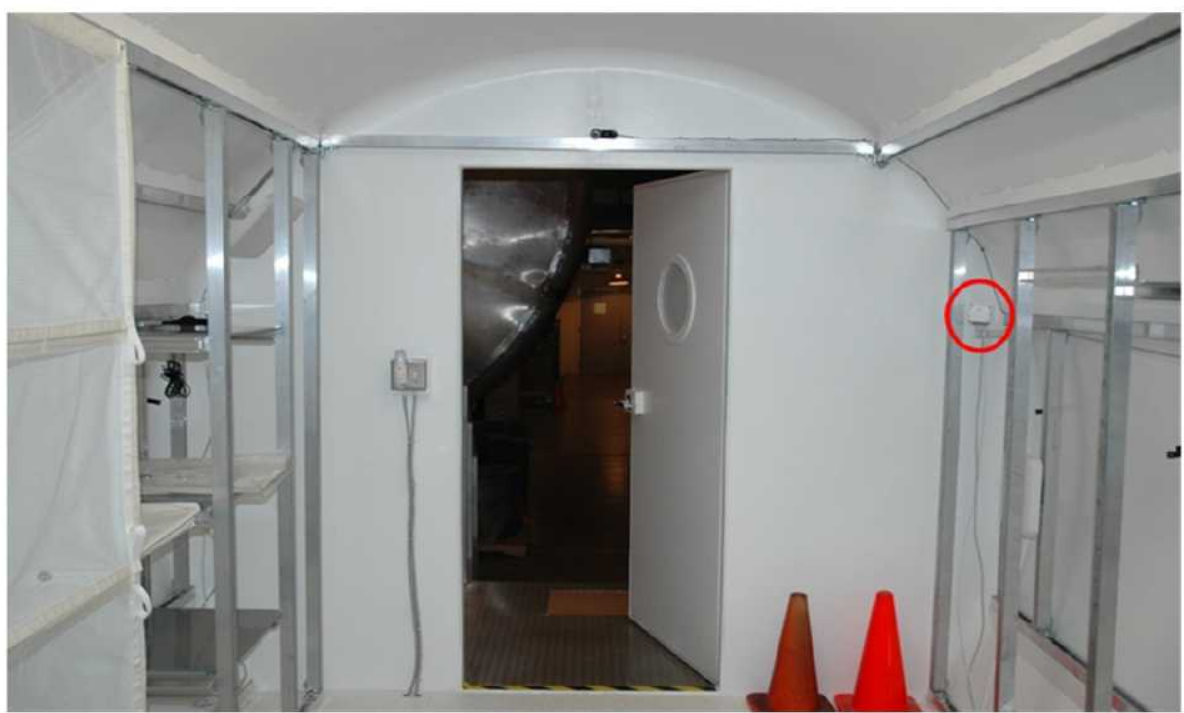

Figure 8. UWB receiver mounted in LHWT. 


\section{Surface Acoustic Wave (SAW) RFID and Space Applications}

\section{A. SAW RFID Background}

In addition to IC-based RFID tags, another type of passive RFID tag is made possible by surface acoustic wave (SAW) technology. Although the market for this type of passive RFID is a small fraction of IC-based RFID market, the technology is capable of satisfying certain niche requirements for NASA.

For example, SAW RFID tags do not need to rectify incident electromagnetic power arriving at the tag. Instead, they modulate and re-radiate the interrogation signal directly using a series of reflectors printed on the surface of the device. As a result, they are capable of operating at much lower interrogator transmit power, and/or are capable of much greater range for a given transmit power than IC-based tags. In addition, SAW RFID tags have the capability to incorporate certain types of sensor telemetry along with the identification information. For example, the impulse response of a SAW RFID tag is highly sensitive to temperature. The temperature of the tag can be estimated directly by using a correlation operation to measure the time dilation (or contraction) of the impulse response. Finally, SAW RFID tags are very robust and can tolerate extreme conditions that would render IC-based tags inoperable. For example, they can withstand temperatures from cryogenic to over $310 \mathrm{C}$, they are inherently immune to ionizing radiation, and they have survived shock levels exceeding $1000 \mathrm{~g}$.

\section{B. SAW RFID for Space-Based Applications}

To exploit these features, SAW RFID technology is being investigated for space-based applications, particularly to support planetary surface operations. Examples include interrogation of sensors on the exterior of a habitat module, interrogation of lunar road signs or "breadcrumbs", location of expended or surplus equipment in a planetary salvage yard, tracking crew and vehicles in the habitat proximity, and interrogation of passive navigation aids for lunar landers. A SAW-based RFID system called the Passive Adaptive RFID Sensor Equipment (PARSEQ) system was integrated with the habitat test bed networks and has been extensively tested for long-range tag interrogation as well as temperature sensing and tag location estimation. The PARSEQ system is illustrated in Fig. 11 and Fig. 12 below.

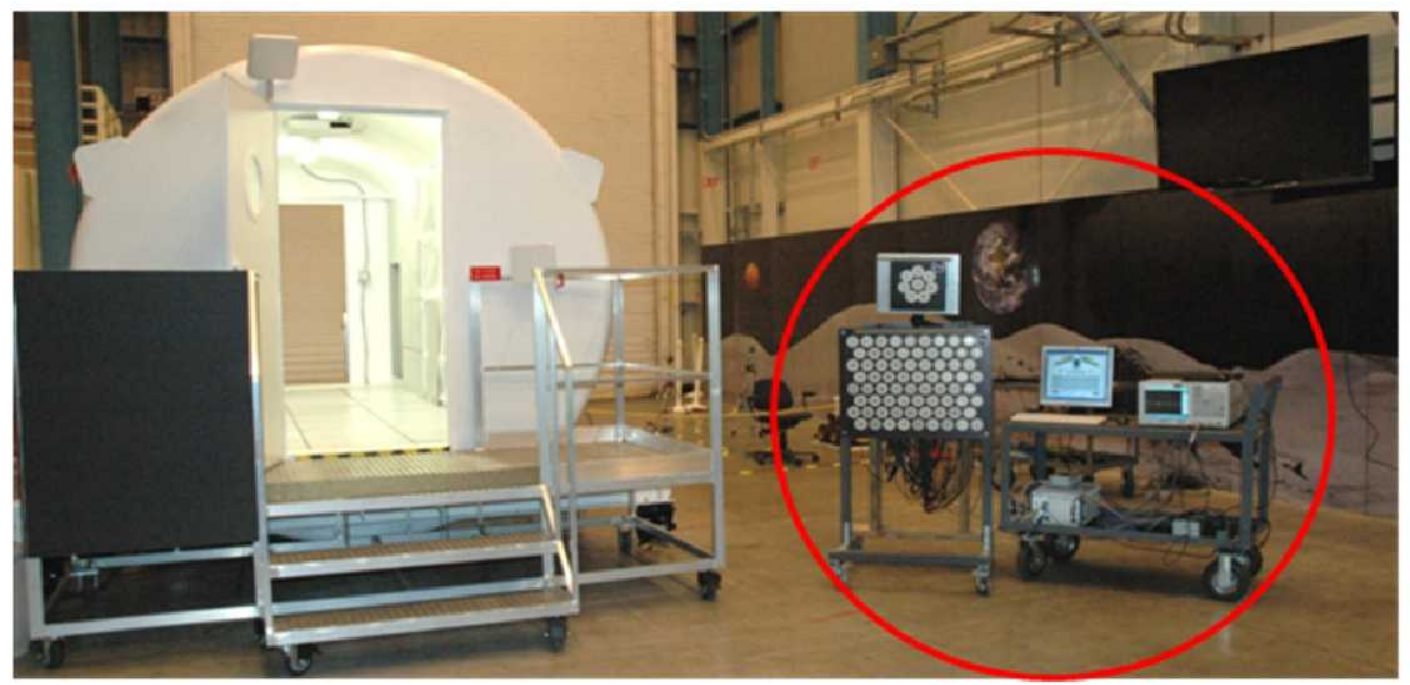

Figure 11. Image of PARSEQ system (circled in red) deployed next to LHWT. 


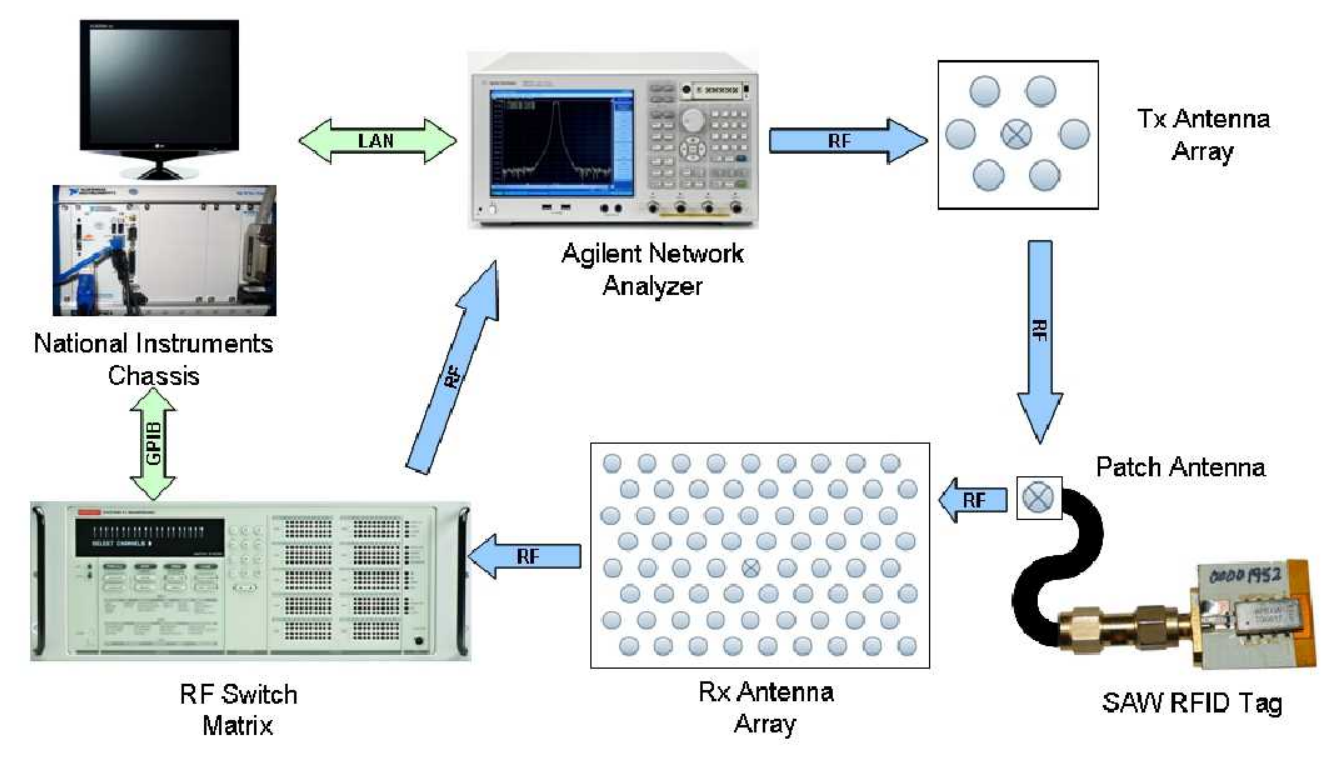

Figure 12. Hardware architecture for PARSEQ system.

\section{BioNet Middleware}

The NASA Constellation Program $(\mathrm{CxP})$ is responsible for building the systems that will return humans to the Moon and then onto Mars. Returning humans to explore and inhabit the lunar surface for long-duration periods necessitates a change in system design philosophy and architecture since (1) the missions are no longer just a few days; and (2) reusable systems will be required to support long-duration human exploration of a planetary surface. This is a significantly different time-scale of mission operations and lifetime expectation of attendant support systems.

The time horizon for the short-duration sortie missions associated with Apollo-era and Shuttle-era missions promoted a system design mentality to accomplish the current mission without a view towards supporting follow-on missions. In this scenario, the design engineers attempt to minimize cost and complexity while meeting mission requirements for just a single mission as opposed to multi-mission support spanning numerous years.

In contrast, for long-term lunar habitat operations, it is reasonable to expect that (1) both wired and wireless data-producing hardware (e.g., for inventory control or environmental control) will be utilized; and (2) that all hardware required for mission operations will not be known a priori, necessitating the requirement for ease-ofadding new hardware to an existing lunar habitat command and control system.

The NASA Command, Control, Communications and Information (C3I) Interoperability Specification (NASA CxP 70022, Vols. 1 - 7) has been proposed as an architectural approach to enable the "complex orchestration of major new human and robotic vehicles and ground systems which are developed by many different sources" [1]. BioNet, a preliminary instantiation of the C3I architecture, is deployed in the NASA Lunar Habitat Wireless Test Bed (LHWT)

The BioNet middleware and software framework is utilized by the LHWT to (1) integrate wired and wireless data producing command and control hardware; and (2) to provide an application development framework to enable independent development of monitor and control user applications that are consumers of acquisition and control system data. With BioNet, the focus is on enabling a "system of systems" and the ability to evolve while meeting spaceflight software certification constraints.

The BioNet middleware provides publish/subscribe asynchronous messaging between distributed applications and distributed data-generating endpoint sensors or systems. The BioNet system architecture provides the necessary infrastructure to facilitate the incorporation of heterogeneous wired and wireless sensing and control devices into a unified data system with a standardized application interface. A key advantage of the BioNet architecture is the focus on the application developer to enable composition of complex applications across multiple heterogeneous node networks. 
The BioNet middleware provides many capabilities in addition to simple management of data producers and data consumers. Middleware is an enabling technology for complex distributed systems. It provides a software development framework, standard services, and network communications for distributed applications. A properly designed middleware eases software development for the application programmer by providing standardized services, coded once, for reuse in multiple applications. Conceptual design integrity is facilitated and maintained since system applications are composed within a common framework. The complexity of network communications, across possibly multiple heterogeneous networks, is hidden from application developers who simply read and write data with no regards to lower-layer communications specifics. In the BioNet system architecture, the middleware layer takes on the greatest importance by providing critical integrated system services including naming, device discovery, service discovery, security, data compression, and data grouping as shown in Fig. 13. Ultimately, the BioNet middleware provides improved information accessibility, which leads to increased situational awareness and improved decision-making capabilities.

The BioNet Developer Kit is provided as open source software to independent developers so that any vendor device can be integrated into the system. The "Dev Kit" provides a C source code module that uses standard Clanguage libraries that serve as a detailed example for any developer. In addition to C-language bindings, Perl and Python bindings are also available.

BioNet was utilized within the LHWT for (1) the integration of various inventory management hardware, including handheld and portal-based RFID systems, "smart" shelves, and the "smart" receptacles for RFID asset tracking. BioNet was ported to the Apple iPod/iPhone for the NASA LHWT project, thereby providing a mobile hand-held command and control device (see Fig. 14). BioNet will easily allow new communication protocols, either standards based (e.g., ISA100) or proprietary, to be added to the distributed network control system and enable the integration of new inventory management hardware (e.g., SAW-based sensors) into the unified asset management control system. BioNet's features were developed in collaboration with NASA engineers to provide the proven benefits of data interoperability, elimination of vendor lock-in, independent developer support, wireless mobility, network scalability, and network robustness and reliability. See Appendix for a complete list of BioNet features and benefits.

In addition to supporting the Lunar Habitat inventory management system, BioNet is deployed on the CGBA-4 and CGBA-5 payloads onboard the International Space Station providing payload command, control and communications in a spaceflight environment. BioNet additionally provides disruption tolerant networking (DTN) communications enabling the transition from a mission-by-mission point-to-point communications architecture to a true multi-hop space network (the Interplanetary Internet).

BioNet reduces cost and risk by directly enabling interoperability (a common goal of middleware) and reduces overall system complexity by providing common services (e.g., security, quality of service, device discovery, etc.) in middleware - coded once and reused. This design directly minimizes flight software certification costs which often are the primary software development cost for NASA. BioNet, as a middleware, can either replace or exist in parallel with current data acquisition and communication systems, easing the transition from a significant installation base of legacy systems. Together, BioNet and DTN improve data timeliness associated with robotic and human-tended missions, assisting NASA in reducing risk, reducing cost, increasing crew safety, improving operational awareness, and improving science return. 


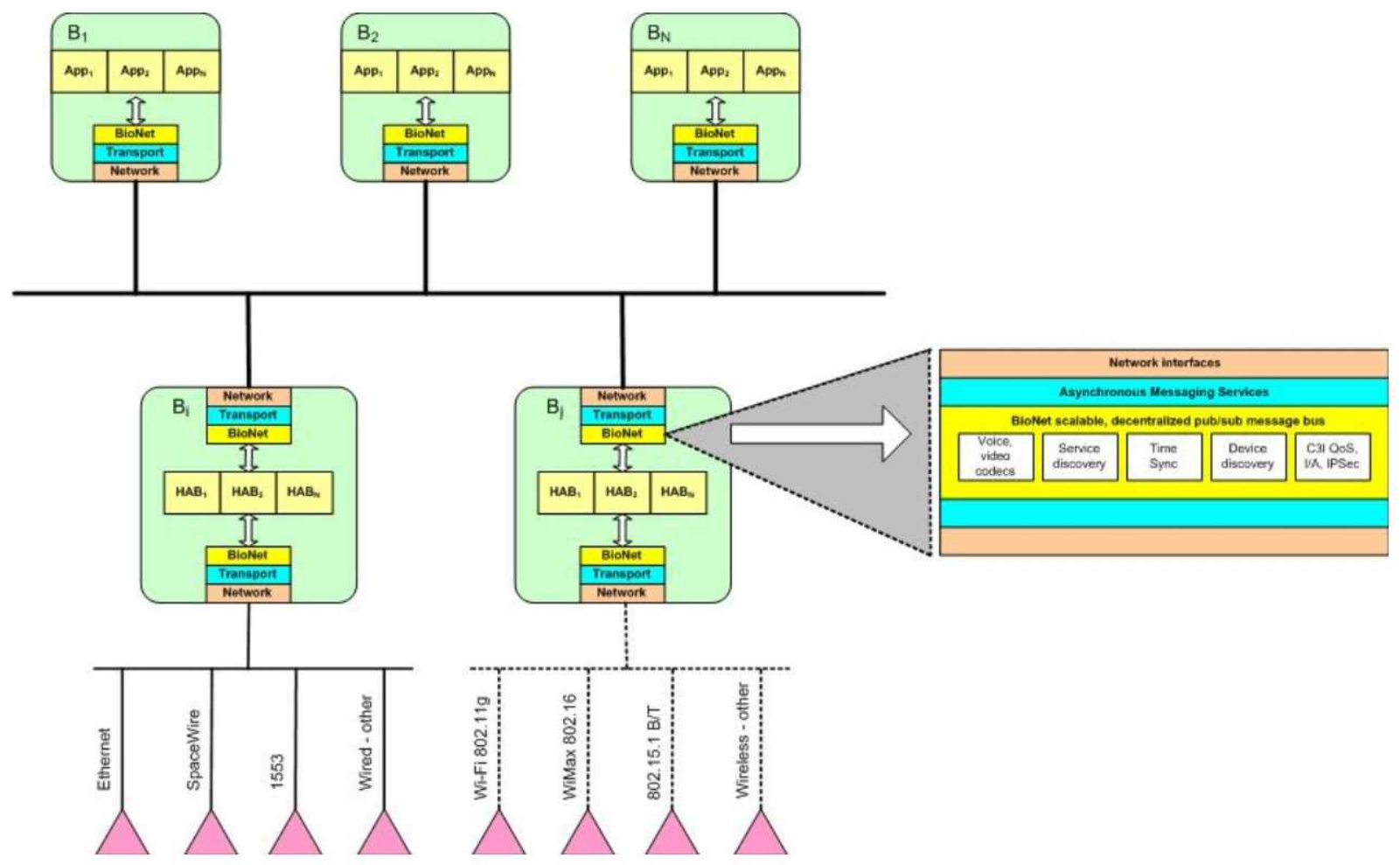

Figure 13. The BioNet distributed peer-to-peer network architecture. Hardware abstractors (HABs) are responsible for abstracting the communication complexity of heterogeneous devices and providing the BioNet data normalization that enables very-easy-to-compose command and control applications.

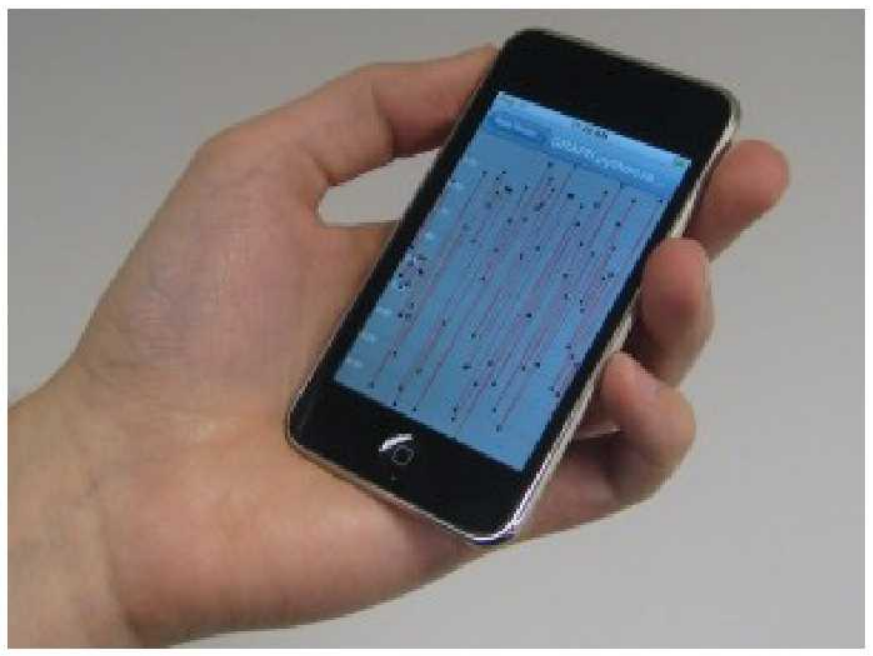

Figure 14. BioNet parameter graphing and monitoring via an iPod. 


\section{Appendix}

\begin{tabular}{|c|c|}
\hline Benefit & Feature \\
\hline Data interoperability & $\begin{array}{l}\text { BioNet integrates data from disparate data-producing sensors, controllers. Voice, video and data } \\
\text { can be stored, transported and display by a single unified control and communications system. }\end{array}$ \\
\hline $\begin{array}{l}\text { Multi-vendor solution } \\
\text { set }\end{array}$ & $\begin{array}{l}\text { Multi-vendor hardware (instrumentation, controllers, radios, etc.) can be considered for the } \\
\text { engineering solution. Non-proprietary (open) as well as proprietary solutions can both be } \\
\text { integrated within the BioNet enterprise software framework. }\end{array}$ \\
\hline $\begin{array}{l}\text { Independent } \\
\text { development }\end{array}$ & $\begin{array}{l}\text { The BioNet framework and Development Kit (DevKit) enables system software development by } \\
\text { multiple independent software developers of: (1) software to interface with hardware devices and } \\
\text { (2) client applications that can make use of any piece of system data for decision-making. }\end{array}$ \\
\hline Mobility & $\begin{array}{l}\text { BioNet is explicitly designed for seamless roaming, enabling untethered mobility of sensors, instrumentation } \\
\text { equipment, communications devices, and personnel for maximum productivity. }\end{array}$ \\
\hline $\begin{array}{l}\text { Standards-based } \\
\text { interoperable network } \\
\text { communications }\end{array}$ & $\begin{array}{l}\text { Allow for interoperable use of differing radio (RF) technologies along with traditional wired data transmission for } \\
\text { maximum flexibility [RFID, IEEE 1902.1 (Rubee), 802.11 (Wi-Fi), 802.15.1 (Bluetooth), 802.15.4 (ZigBee), } \\
802.16 \text { (Wi-Max), 802.22 (WRAN), IEEE 802.3, USB and serial]. }\end{array}$ \\
\hline Scalability & $\begin{array}{l}\text { The BioNet peer-to-peer distributed architecture enables highly scalable systems from tens of devices to } \\
\text { thousands of data-producers enabling true 'systems of systems' scalability. }\end{array}$ \\
\hline $\begin{array}{l}\text { Network robustness } \\
\text { and reliability }\end{array}$ & $\begin{array}{l}\text { The BioNet peer-to-peer architecture automatically reconfigures to enable entire subnetworks to enter or leave } \\
\text { the communications and control network. The peer-to-peer architecture keeps operating in the face of network } \\
\text { partitions and is self-healing to allow networks to recombine. }\end{array}$ \\
\hline Platform independence & BioNet adheres to open-architecture and provides standards-based solutions for maximum interoperability. \\
\hline $\begin{array}{l}\text { Ease of application } \\
\text { composition }\end{array}$ & $\begin{array}{l}\text { BioNet provides helper libraries and services to provide unified interface to device and service discovery, time } \\
\text { synchronization, security, compression, etc. The middleware acts as the "service broker" offering a set of } \\
\text { services with defined interfaces for ease of implementation. }\end{array}$ \\
\hline $\begin{array}{l}\text { Eases burden of flight } \\
\text { software certification }\end{array}$ & $\begin{array}{l}\text { The BioNet framework is designed to provide standardized services for application developers, easing the } \\
\text { software development process and ensuring conceptual integrity of the system. }\end{array}$ \\
\hline $\begin{array}{l}\text { Unified enterprise data } \\
\text { management system }\end{array}$ & $\begin{array}{l}\text { BioNet integrates future and legacy heterogeneous devices into a common system based on an open } \\
\text { architecture concept that supports widely recognized IEEE standards, as well as supporting vendor-specific } \\
\text { (proprietary) protocols for unique applications. }\end{array}$ \\
\hline $\begin{array}{l}\text { Automated data } \\
\text { replication and back-up }\end{array}$ & $\begin{array}{l}\text { Provide automated database synchronization mechanisms that synchronize data stores as BioNet nodes move } \\
\text { dynamically in and out of the network. }\end{array}$ \\
\hline $\begin{array}{l}\text { Consistent operational } \\
\text { interface }\end{array}$ & $\begin{array}{l}\text { Abstracting networks and hardware for the application developer simplifies composition of common command, } \\
\text { control and monitoring tools. This directly results in crew time minimization. }\end{array}$ \\
\hline $\begin{array}{l}\text { Integrated network } \\
\text { security }\end{array}$ & BioNet provides integrated security services for data privacy, data integrity, authentication and authorization. \\
\hline $\begin{array}{l}\text { Delay tolerant } \\
\text { communications }\end{array}$ & $\begin{array}{l}\text { BioNet provides reliable message transfer across high-latency, intermittently-connected links enabling } \\
\text { networked interplanetary communications. }\end{array}$ \\
\hline $\begin{array}{l}\text { Engineering data } \\
\text { analysis }\end{array}$ & $\begin{array}{l}\text { BioNet provides a historical database for parameter trending evaluation with easily customizable user displays } \\
\text { for optimal graphical visualization. }\end{array}$ \\
\hline $\begin{array}{l}\text { Easy support of add-on } \\
\text { or retro-fit activities }\end{array}$ & $\begin{array}{l}\text { BioNet facilitates the late or retro-fit addition of additional sensors, controllers, and communications equipment } \\
\text { to add additional required functionality as test and verification activities impose new requirements or new } \\
\text { capabilities are deemed necessary. }\end{array}$ \\
\hline $\begin{array}{l}\text { Endpoint re- } \\
\text { programmability }\end{array}$ & $\begin{array}{l}\text { BioNet enables endpoint devices (sensors, radios, etc.) to be selectively chosen and dynamically } \\
\text { reprogrammed to upgrade functionality while maintaining nominal operation of the control system. }\end{array}$ \\
\hline $\begin{array}{l}\text { Provides NASA C3I- } \\
\text { compliant services }\end{array}$ & $\begin{array}{l}\text { BioNet provides NASA Command, Control and Communications Infrastructure (C3I) security, compression, } \\
\text { Quality of Service, Information Architecture, along with standardized Command and Data Exchange services. } \\
\text { BioNet is a complete end-to-end C3I compliant system. }\end{array}$ \\
\hline
\end{tabular}


Table A-1: BioNet middleware benefits and features.

\section{Acknowledgments}

This work was sponsored by NASA's Innovative Partnerships Program.

\section{References}

${ }^{1}$ Rader, S., Kearney, M., McVittie, T. and Smith, D., "Moving Towards a Common Ground and Flight Data Systems Architecture for NASA's Exploration Missions", AIAA 2006-5723, Space Ops 2006. 